Voix et Images

voixetimages

\title{
Concupiscence et avarice chez Séraphin Poudrier
}

\section{Claude-Marie Gagnon}

Volume 1, numéro 2, décembre 1975

\section{Raoul Duguay}

URI : https://id.erudit.org/iderudit/290075ar

DOI : https://doi.org/10.7202/290075ar

Aller au sommaire du numéro

\section{Éditeur(s)}

Les Presses de l'Université du Québec

ISSN

0318-9201 (imprimé)

1705-933X (numérique)

Découvrir la revue

Citer cet article

Gagnon, C.-M. (1975). Concupiscence et avarice chez Séraphin Poudrier. Voix et Images, 1(2), 196-205. https://doi.org/10.7202/290075ar d'utilisation que vous pouvez consulter en ligne.

https://apropos.erudit.org/fr/usagers/politique-dutilisation/ 


\section{Concupiscence et avarice chez Séraphin Poudrier}

Depuis la parution du roman Un homme et son péché, en 1933, le personnage de Séraphin Poudrier a été la cible de nombreux critiques ${ }^{1}$. Ces fougueux polémistes ont été unanimes à voir en Poudrier l'incarnation d'un "péché ": l'avarice.

L'histoire est simple, unie, d'un trait pur et rapide. Séraphin Poudrier n'est pas un avare, il est l'avarice même. L'homme et son péché s'identifient au point que Poudrier existe, et avec quelle intensité ! uniquement par son péché 2 .

Mais il semble qu'on ait négligé un côté important de la personnalité de Séraphin: la luxure. Gérard Bessette, un des seuls à avoir fait une analyse valable de ce personnage, se demande si on n'a pas trop insisté sur l'avarice, au détriment de la concupiscence:

Certes, l'avarice constitue le trait le plus frappant de Séraphin. Non seulement ce vice finit-il par triompher, mais son hégémonie n'est même pas menacée sérieusement au cours de l'intrigue. Toutefois la concupiscence ou, comme Grignon préfère l'appeler, "l'impureté " ou la «luxure» ne laisse pas de montrer de la tête. Et je me demande si ce deuxième trait, ce deuxième penchant - précisément parce qu'it est refoulé, frustré - n'est pas plus significatif que l'avarice pour qui veut comprendre en profondeur la psyché de Séraphin ${ }^{3}$.

Sur ce point, Bessette a entièrement raison. Il est impossible de faire une étude cohérente de Séraphin en laissant de côté le pôle lubrique de sa personnalité. Cependant, là où Bessette fait fausse route, c'est en niant le côté infantile de l'avare: "S'il n'est peut-être pas arbitraire de parler chez lui de "régression" ni même, en un sens, de "fixation maternelle, il me paraît erroné de le situer à un «stade infantile du développement de la

1. Parmi les plus connus, il convient de citer Louis Dantin, Rex Desmarchais, Albert Laberge et M.-A. Lamarche.

2. Rex Desmarchais, "la Vie et la mort de Séraphin Poudrier", dans la Nouvelle Relève, mai 1942, p. 493.

3. Gérard Bessette, "Autour de Séraphin", dans Une littérature en ébullition, Montréal, Jour, 1968, p. 92. 
personnalité" "." Pourtant, le comportement de Séraphin ne peut s'expliquer par une fixation (et non une régression) aux stades oral et anal, comme nous tenterons de le démontrer.

\section{LUXURE ET ORALITÉ CHEZ SÉRAPHIN}

Avant de voir comment luxure et oralité s'entremêlent chez Séraphin, il semble pertinent de définir le stade oral. Selon Freud, qui distingue quatre phases dans la libido (les phases orale, anale, phallique et génitale), la phase orale est la première organisation sexuelle prégénitale. II note que "l'activité sexuelle, dans cette phase, n'est pas séparée de l'ingestion des aliments, la différenciation des deux courants n'apparaissant pas encore ${ }^{5}$." Plus explicites, à notre avis, Laplanche et Pontalis décrivent le stade oral comme le

premier stade de l'évolution libidinale: le plaisir sexuel est alors lié de facon prédominante à l'excitation de la cavité buccale et des lèvres qui accompagne l'alimentation. L'activité de nutrition fournit les significations électives par lesquelles s'exprime et s'organise la relation d'objet; par exemple, la relation d'amour à la mère sera marquée par les significations manger, être mangé 6 .

A la lumière de ces faits, nous pouvons déjà expliquer plusieurs bizarreries du comportement de Séraphin. Citons d'abord le passage où Grignon raconte les fréquentations de l'avare et de Donalda:

II connut Donalda, enfant. II la convoitait depuis le jour où il l'avait rencontrée dans un champ de fraises. Elle s'était assise près de lui et il avait été frappé par la blancheur de ses bras et par la fermeté de sa poitrine, si opulente pour son âge. II l'aimait. [...] Quand la petite eut vingt ans, il l'épousa. II en avait quarante. Les troubles de la chair qu'il combattait depuis tant d'années l'envahissaient maintenant ainsi qu'une crue prodigieuse de limon. Mais Séraphin ne se laissa point attendrir comme un fol, ni par le cœur, ni par les sens. Il se rendit compte avec une précision d'usurier que s'il se laissait aller à la passion de la chair, la petite Donalda Laloge finirait par lui coûter les yeux de la tête et lui mangerait jusqu'à la dernière terre du rang. (Un homme et son péché, Sainte-Adèle, Éditions du Grenier, 1972, p. 12-13).

On constate d'abord que le principal attrait de Donalda est son abondante poitrine. L'avare identifie sans aucun doute la poitrine de Donalda aux seins maternels gonflés de lait, qui lui ont peut-être manqué jadis. Le roman ne donne aucune indication sur l'enfance de Poudrier, mais il est fort possible que Séraphin ait désiré le sein maternel absent, et se soit imaginé la satisfaction qui découle de l'allaitement. De cette frustration serait né un

4. Gérard Bessette, op. cit., p. 91-92. L'auteur commente alors une opinion de Sœur Sainte-Marie-Eleuthère dans la Mère dans le roman canadien-français, Québec, PUL, 1964, p. 49.

5. Sigmund Freud, Trois essais sur la théorie de la sexualité, Paris, Gallimard, "ldées", 1971, p. 95.

6. Jean Laplanche et J.-B. Pontalis, Vocabulaire de la psychanalyse, Paris, P U F, 1968, p. 457. 
sentiment de haine envers ce "mauvais sein" qui refuse de satisfaire l'enfant au moment voulu par ce dernier. Comme l'explique Mélanie Klein:

Les fantasmes primitifs qui accompagnent les sentiments du bébé sont de nature variée. Dans celui que je viens de mentionner, le bébé imagine la satisfaction qui lui manque. Des fantasmes agréables accompagnent la satisfaction réelle tandis que des fantasmes destructeurs sont associés à la frustration de haine qu'elle éveille. Lorsque le bébé se sent frustré par le sein, il attaque ce sein dans ses fantasmes; si le sein le satisfait, il éprouve de l'amour pour lui et il a, dans sa relation avec lui, des fantasmes de nature plaisante. Dans ses fantasmes agressifs, il souhaite mordre et déchirer sa mère et ses seins et la détruire aussi par d'autres moyens ${ }^{7}$.

De plus, le jeune enfant qui craint d'être désintégré par la pulsion de mort qui reste en lui peut projeter son angoisse sur le «mauvais sein": le bébé craint que son attaque du mauvais sein entraîne une réplique de ce dernier. Le nourrisson redoute alors d'être déchiré ou avalé par ce mauvais sein, puisqu'au stade oral il ne peut imaginer une autre forme de danger.

Les amours entre Donalda et Séraphin reproduisent de façon frappante ce fantasme de la phase orale. Comme on l'a vu précédemment, Séraphin désire "engloutir" Donalda. Il se laisse d'abord entraîner par sa passion, mais les vieux fantasmes de l'enfance remontent à la surface: il craint d'être déchiqueté et avalé. En effet, l'avare dit de Donalda qu'elle "finirait par lui coûter les yeux de la tête et lui mangerait jusqu'à la dernière terre du rang". Les yeux représentent les organes génitaux, comme l'explique Sandor Ferenczi :

C'est ainsi que la moitié supérieure du corps, tenue pour plus anodine, acquiert sa signification symbolique et que se réalise ce que Freud a appelé "le déplacement du bas vers le haut". Dans ce travail du refoulement, les yeux, du fait de leur sensibilité et de leur grande valeur, se sont révélés particulièrement aptes à accueillir les affects déplacés des organes génitaux ${ }^{8}$.

Et Séraphin éprouve une peur qui s'apparente à la castration, mais qui est en réalité une peur orale, une des Sept peurs infantiles décrites par Bergler: «Je crois que l'enfant peine sous le fardeau des Sept peurs infantiles et qu'il vit entouré des terreurs suivantes: Peur de mourir de faim, d'être dévoré, empoisonné, étouffé, coupé en morceaux, vidé, châtré. [...] Une grande partie de ce qui se cache sous la peur de la castration est au fond une peur orale non-éliminée, dérivée des Sept peurs ${ }^{9}$.»

En plus, il y a le fameux verbe «manger» accolé à Donalda. On imagine mal comment une femme si frugale et si soumise pourrait ruiner Poudrier. En fait, Séraphin projette en Donalda sa propre agressivité. La jeune femme est vue comme un danger, parce qu'elle est celle qui engloutit.

7. Mélanie Klein, "l'Amour, la culpabilité et le besoin de réparation », dans l'Amour et la haine, Paris, Payot, “Petite Bibliothèque Payot ", i 968, p. 79-80.

8. Sandor Ferenczi, "le Symbolisme des yeux", dans CEuvres complètes, t. II, Paris, Payot, 1968, p. 69.

9. E. Bergler, "Symptômes et signes de la régression orale", dans la Revue française de psychanalyse, t. XXIV, $n^{\circ} 1,1960$, p. 60-61 et 64 . 
La seule issue, pour l'avare, est de lutter contre la concupiscence, sous peine d'être dévoré: «Et si les flammes de la luxure s'acharnaient sur Séraphin, l'homme alors les combattait comme il pouvait. » (p. 14)

Justement, Séraphin a bientôt l'occasion de voir ce qui lui en coûterait de succomber à la luxure. En effet, peu après ce passage où Grignon relate les fréquentations du couple Poudrier, l'avare reçoit la visite de JeanBaptiste Lemont, un emprunteur (car en plus d'être cultivateur, Poudrier prête à usure). Ce Lemont, qui semble n'avoir aucune utilité dans le roman, est en réalité le "double sombre ${ }^{10}$ " de Séraphin. Car Lemont est l'incarnation parfaite de cette luxure que Poudrier tente de combattre: il a engrossé la petite Célina Labranche, «une paysanne de quinze ans » (p. 48), sans doute le même âge que Donalda lorsqu'elle excita Séraphin. Pour Lemont comme pour Poudrier, il s'agit "d'une seule fois" et de brutalité 11: "Lemont ayant attiré la petite Célina dans la grange, l'avait quasiment prise de force." (p. 34) Quant à Donalda, “Séraphin la posséda brutalement [...] une fois, une seule fois". (p. 14) L'intention de l'avare est nette: il veut châtier Lemont, tout en se punissant à travers lui : "ll avait maintenant devant lui le monstre d'impureté. L'avarice opiniâtre ferait payer cher à la luxure ses joies fugitives". (p. 34) Et la punition de Lemont est une punition orale: Séraphin le "sèvre" en lui retirant ses deux vaches jerseys, celles qui donnent le lait «le plus riche de la paroisse» (p. 172), et qui sont évidemment les deux seins maternels, la possession la plus chère de l'enfant. A ce stade, on ne peut guère imaginer pire châtiment.

Cependant les tendances lubriques de Séraphin ne sont qu'apaisées.

Sa cousine Bertine vient bientôt se substituer à Donalda malade, qui a perdu tous ses charmes pour l'avare. La Donalda de jadis et Bertine sont interchangeables. Elles sont jumelles par le teint: la première est "fraîche comme un pommier en fleur» (p. 13), la seconde a "un teint de pomme mûre" (p. 65), et par les seins: Donalda possède une poitrine opulente et celle de Bertine "la plus belle du monde, [...] faisait éclater le corset trop petit". (p. 65) Mais Bertine est, elle aussi, un fruit défendu pour l'avare. En plus d'être la fille de son cousin Alexis, elle représente également le "mauvais sein". Alors que Donalda constituait un péril pour l'avare uniquement s'il se laissait aller à la luxure, le seul fait de songer à posséder Bertine est un danger pour Séraphin : "ll imaginait les moyens de posséder bientôt, tout à l'heure, dans le foin de la grange, cette ragoûtante paysanne aux lèvres charnues et rouges, à l'œil vif où paraissaient courir des lueurs

10. Le double est une figure qui vient rappeler au mol tout ce qu'll a voulu, par un mécanisme de défense, rejeter hors de lui. Dans son étude sur Racine, Mauron définit le double comme "la moitié de la personnalité qui a été refoulée par l'autre mais lui demeure vitalement liée et la poursuit comme son ombre". (L'Inconscient dans l'œuvre et la vie de Racine, Aix-en-Provence, Faculté des lettres, éd. Ophrys, 1957, p. 33) Et tel est bien le rôle joué par l'homme roux.

11. La brutalité, ou le sadisme, est une des caractéristiques de la phase prégénitale, ainsi que le signale Freud: "In the development of the libido in man, the phase of genital primacy must be preceded by a 'pregenital organisation' in which sadism and anal erotism play the leading part". Collected Papers, vol. II, Londres, Hogarth Press, 1956, p. 165. 
de convoitise et d'abandon. » (p.68) Ceci entraîne une réaction de la part de la jeune fille, et l'avare perçoit cette réaction comme une agression. »

Mais il fut arraché à sa rêverie par la présence de la jeune fille qui se tenait devant lui, un couteau à la main.

- Quel poulet qu'on va tuer, cousin, demanda-t-elle?

- Hein. Tuer une poule! Tuer une poule! répéta-t-il.

Mais il se ressaisit aussitôt:

- T'as raison. J'y vais justement pensé, moi itou. J'allais justement en tuer une avant que t'arrives. (p. 69)

Notons que la volaille change de sexe d'une réplique à l'autre, comme l'a fait remarquer Bessette. Bertine veut tuer «un poulet»; Séraphin, affolé, répète: "tuer une poule", et se ressaisit seulement ensuite. Bessette y voit une "peur de la castration 12 ". Nous ne croyons pas qu'on doive y voir le fameux complexe de castration, qui n'apparaît qu'au stade phallique, mais plutôt une des “peurs infantiles" décrites précédemment par Bergler: la peur d'être coupé en morceau et vidé de sa substance comme le poulet. N'oublions pas que Séraphin conçoit tout danger et toute forme d'agression du point de vue oral. L'avare s'identifie à ce poulet, et le couteau de Bertine est une arme meurtrière. Car Séraphin, qui assimile la jeune fille à Donalda, donc au " mauvais sein ", a projeté en elle ses propres pulsions de mort.

En fait, Séraphin est tellement ébranlé qu'il abandonne tout projet de fornication et se rabat sur le voyeurisme ${ }^{13}$.

S'il pouvait voir Bertine se déshabiller. Seulement l'entrevoir. Un éclair de peau dans la nuit. La durée d'un instant. Ah! combien il garderait toute sa vie, dans ses yeux, cette affolante image. Mais il ne bougeait pas, espérant toujours qu'il entendrait le bruit d'un corset qu'on enlève. [...] Son idée était d'arriver au grenier en deux bonds, et de surprendre Bertine à moitié nue. (p. 77-78)

Mais ce projet ne réussit pas mieux que le précédent. En montant l'escalier branlant qui conduit au grenier, Séraphin fait craquer une marche, ce qui alerte Bertine. Bessette émet l'hypothèse qu'un «acte manqué » soit à l'origine de cette maladresse de Séraphin:

On ne saurait concevoir un plus grand écart entre la représentation ( «son idée était d'arriver au grenier en deux bonds") et l'action («il monta la première marche... il mit le pied sur la troisième marche»). On a affaire à un "acte manqué " au sens freudien du ierme, et il est sûr que Séraphin est soulagé quand la marche craque. Dès que Bertine lui adresse la parole, il se montre penaud comme un enfant pris en faute. C'est proprement l'attitude du garçonnet que sa mère vient de gronder parce qu'il ne voulait pas se coucher.

— «Bonsoir, dit faiblement Séraphin qui décida de se coucher». (p. 78)

12. Gérard Bessette, op. cit., p. 97.

13. Le voyeurisme, comportement actif mais perversion chez l'adulte, est une des composantes de la sexualité infantile et fait son apparition au stade oral. II faut signaler que Séraphin s'adonne au voyeurisme dès que Bertine a tué la fameuse poule. "Quelle fille smatte, pensait Séraphin qui ne la quittait pas des yeux. Et viande à chiens! Quelles fesses itou, quelles fesses." (p. 70) Remarquons que c'est la région anale qui attire l'attention de Séraphin. 
Et ce sera dans un lit d'enfant qu'il va se coucher:

"ll se fabriqua un lit avec deux chaises, des manteaux, et une vieille couverture». (p. 78) ${ }^{14}$

$\mathrm{Ce} q u$ 'il faut retenir ici, c'est que Bertine est assimilée à la mère. Cependant Bessette fait erreur quand il écrit: "Avec Donalda, l'attitude de l'avare sera celle d'un père sévère, inplacable ${ }^{15}$." Peut-être est-ce là une impression produite par l'attitude de Séraphin? Car inconsciemment, nous l'avons vu plus haut, Donalda est «la mère». Consciemment - et il est intéressant de le noter - Donalda n'est considérée comme mère qu'au moment où elle va mourir, c'est-à-dire lorsqu'elle a cessé d'être le «mauvais sein $\approx$ :

- Mon pauvre vieux [dit-elle], (et sa voix était devenue plus brève) promets-moi de jamais te remarier. Tu seras toujours plus heureux tout seul.

- Je te le promets, ma vieille. (p. 90)

et une autre fois, lorsqu'elle vient de mourir:

Séraphin passa deux fois la main sur le front de la morte. II ne fut pas étonné de le sentir déjà froid.

- Paurre vieille, dit-il, t'as ben souffert depuis quelques jours. Tu souffriras pus jamais à c't'heure. (p. 107)

Les tendances orales de Séraphin se manifestent particulièrement en ce qui concerne la concupiscence. Cette dernière est jugée mauvaise par l'avare qui fait tout pour l'éteindre. Cette concupiscence, teintée de gourmandise et de brutalité, est projetée dans le «mauvais sein ». Séraphin ne connaît pas le «bon sein». Tout ce qu'il a pu idéaliser, c'est l'argent, et il a tellement idéalisé le précieux métal qu'il a accédé au stade anal en devenant un avare.

\section{STADE ANAL ET AVARICE}

Laplanche et Pontalis définissent le stade anal comme le

deuxième stade de l'évclution libidinale, selon Freud, qu'on peut situer approximativement entre deux et quatre ans; il est caractérisé par une organisation de la libido sous le primat de la zone érogène anale; la relation d'objet est imprégnée de significations liées à la fonction de défécation (expulsion-rétention) et à la valeur symbolique des fèces ${ }^{16}$.

Freud, de son côté, a décrit les trois particularités du caractère anal comme étant «l'ordre, la parcimonie - qui peut être exagérée au point de devenir l'avarice - et l'obstination ${ }^{17}$ ".

14. Gérard Bessette, op. cit., p. 99.

15. Ibid.

16. Jean Laplanche et J.-B. Pontalis, op.cit, , p. 460.

17. Sigmund Freud, Collected Papers, t. 2, p. 45. 
L'avarice de Séraphin Poudrier est d'abord caractérisée par la rétention: il refuse catégoriquement de faire le moindre don. II consent à prêter, mais à des taux si exorbitants, que cela lui permet d'augmenter son capital. Toutefois, il conserve la majeure partie de son argent dans l'un des trois sacs d'avoine du haut-côté.

Cette rétention de l'argent est semblable à la rétention des fèces pour l'enfant. En effet, Groddeck voit dans la rétention des matières fécales une intervention du ça qui veut conserver pour lui ce qui devrait être remis au monde extérieur. Il distingue trois raisons qui poussent l'enfant à retenir ses excréments :

En général, on peut dire qu'une des trois motions psychiques y participe le plus souvent: le ça peut estimer: "Le monde extérieur n'est pas digne que je lui communique mon intérieur"; ou encore: "J'ai tant de plaisir a cette rétention que les droits du monde extérieur n'entrent plus en ligne de compte"; ou enfin: "Le contenu de mon intérieur est si médiocre que j'ai honte de le donner au monde extérieur ${ }^{18}$.»

En ce qui concerne Séraphin, c'est la deuxième motion qui dicte son attitude. Au début du roman, nous apprenons que Séraphin refuse un enfant à Donalda: "[II] refusa net de lui faire un fils qu'elle désirait avec tant d'amour, de par l'hérédité la plus lointaine.» (p. 14) Pourquoi ne se satisfait-il qu'une seule fois avec Donalda? A-t-il peur d'avoir des enfants? Certes pas, puisque le grippe-sou sait pratiquer le coït interrompu. Donalda se serait-elle refusée à cette pratique? De la part de "cet être faible, timide, sans volonté aucune ${ }^{19}$ ", c'est plutôt improbable. Cette attitude de Séraphin a dû causer bien des maux de tête à Gérard Bessette qui a escamoté presque complètement le problème: "Mais alors, pourquoi Séraphin, s'il sait se satisfaire sans danger de progéniture, ne possède-t-il sa femme "qu'une fois, une seule fois ${ }^{20}$." La "seule fois", il a satisfait le côté sadique de sa personnalité, comme on l'a signalé précédemment. De plus, la conduite de Séraphin s'explique par son caractère anal qui refuse absolument le don: don du sperme d'abord, don de l'argent ensuite, pour vêtir et nourrir ses enfants.

L'avare ne peut se résoudre à se départir de l'argent. La bourse enfermée dans le sac d'avoine, comme les fèces à l'intérieur de l'enfant, est une source inépuisable de plaisir:

Dans un des sacs, l'usurier cachait une grande bourse de cuir ne renfermant jamais moins de cinq cents à mille dollars en billets de banque, en pleces d'argent, d'or et de cuivre. II ne déposait pas toujours la bourse dans le même sac. Mais il savait positivement, absolument dans lequel des trois il l'avait mise. Alors il le regardait avec amour, puis marmonnait de vagues paroles. Une curiosité immense, suivie d'une sensation inexprimable, s'emparait de lui, coulait dans tout son

18. Georg Groddeck, "la Constipation comme résistance type», dans la Maladie, l'art et le symbole, Paris, Gallimard, 1969, p. 115.

19. Albert Laberge, Peıntres et écrivains d'hier et d'aujourd'hui, Montréal, 1938, p. 177

20. Gérard Bessette, op. cit., p. 95. 
être ainsi qu'une poussée de sang neuf et rapide. C'était trop de félicité: Séraphin ne pouvait plus se retenir. II plongeait sa main osseuse et froide dans le sac. Avec lenteur, avec douceur, il tâtait, il palpait, il fouillait parmi les grains d'avoine, et lorsqu'il sentait enfin ô suprêmes attouchements! - la bourse de cuir ou simplement les cordons, sa jouissance atteignait à un paroxysme que ne connut jamais la luxure la plus parfaite, et son cœur battait, fondait, défaillait. (p. 25-26)

II est évident que Séraphin a transféré sur l'argent son gain de plaisir obtenu par la rétention du contenu intestinal. L'argent lui procure une satisfaction analogue à celle que le nourrisson tire de la défécation. Dans les deux cas, il y a confirmation de la puissance de l'individu. L'enfant tient sa mère, ou la personne qui s'occupe de lui, à sa merci: «If an infant obstinately refuses to empty its bowel when placed on the chamber, that is, when the nurse wishes, but withholds this function at his own pleasure 21 . "Dans le cas qui nous intéresse, l'avarice de Séraphin Poudrier reproduit le même schème: l'argent le rend tout-puissant auprès des autres villageois :

Lui, Séraphin Poudrier, petit cultivateur, petit prêteur de rien du tout, mais qui tenait tout le monde dans sa main: monsieur le maire, monsieur le docteur, monsieur le député, tous les cultivateurs, depuis le plus gros jusqu'au plus petit. Peu d'hommes mangeant et ayant besoin d'un gîte qui ne lui devaient de l'argent. Il était le possesseur et le maître. Une joie profonde, sans limites, bleue comme un ciel de printemps, l'inondait. (p. 25-26)

De plus, comme Groddeck l'a signalé, «l'enfant - et plus généralement l'inconscient de l'adulte - apprend à connaître le concept de propriété par l'inconscient, les excréments se confondant à l'argent, et leur valeur est celle de l'argent ${ }^{22}$."En fait, comme l'explique aussi Ferenczi,

les matières fécales sont les premières économies de l'être en devenir et comme telles restent en corrélation inconsciente avec toute activité physique ou mentale qui a quelque chose à voir avec l'action d'amasser, d'accumuler et d'épargner ${ }^{23}$.

Cependant, à mesure que l'enfant grandit, son dégoût pour les matières fécales augmente ${ }^{24}$. II cherche des objets pour les remplacer. Comme substituts, il y a la boue qui a la même consistance que les fèces; le sable, apparenté aux matières fécales déshydratées; des pierres, des billes de verre, qui constituent déjà une sorte de monnaie primitive. Enfin, le dernier pas est franchi: les fèces sont assimilées à l'argent, qui permet à l'enfant d'obtenir tout ce qu'il désire.

21. Sigmund Freud, Collected Papers, t. II, p. 47.

22. Georg Groddeck, op. cit., p. 123.

23. Sandor Ferenczi, op cit., p. 143.

24. Freud et Ferenczi situent ce dégoût pour les fèces à l'époque où l'enfant commence à marcher: "L'intérêt de l'enfant pour ses déjections subit sa première distorsion du fait que l'odeur des fèces lui devient désagréable et lui répugne. Ceci est probablement en rapport avec le début de la marche verticale». Ferenczi, op. cit., p. 143. 
Pour prouver ce lien, Ferenczi ajoute: “La jouissance liée au contenu intestinal devient plaisir procuré par l'argent, qui n'est rien d'autre que des excréments désodorisés, déshydratés, et devenus brillants ${ }^{25}$."

Mais il faut dire que Séraphin possède un caractère anal, et non un érotisme anal. Ferenczi fait la distinction: "Le caractère anal avec son amour de la propreté et de l'ordre, son entêtement et son avarice, contraste étrangement avec un érotisme anal marqué, qui est tolérant pour la saleté, prodigue et bon ${ }^{26}$." S'il y a un personnage qui possède un érotisme anal dans Un homme et son péché, ce n'est pas Séraphin, mais bien son cousin Alexis, "qui sent la tonne et les femmes» (p. 172) et «dépense comme un fou dans une semaine, tout ce qu'il avait arraché au sein de sa vieille terre» (p. 49). Séraphin, par contre, fait preuve d'un grand souci d'ordre et de propreté. La pì̀ce du haut-côté, repaire de l'avare, est meublée «d'un petit poêle toujours bien propret» (p. 22), et d'un petit secrétaire en acajou où "on ne manquait jamais de trouver du papier réglé, une plume, un crayon". (p. 23) C'est aussi un cultivateur ordonné : "Les récoltes se trouvaient à l'abri, et aussi sur la terre froide, il ne restait pas une seule carotte, une betterave, un seul chou, une seule patate. L'ordre avait marché avec les saisons, et il régnait comme par le passé.» (p. 56) De plus, Séraphin est très opiniâtre lorsqu'il prête à usure:

Le prêteur doublé d'un usurier avait l'habitude dans ses marchés, surtout lorsqu'il s'agissait de faire signer des billets à ordre, de ne jamais parler le premier. II attendait son homme, comme il disait. II observait le moindre de ses gestes. Tout emprunteur était à ses yeux un adversaire extrêmement dangereux; tout débiteur devenait et demeurait à ses yeux un malfaiteur. II valait mieux laisser d'abord cette engeance "s'ouvrir" et la guetter, solide, sur la défensive. (p. 30)

Enfin, pour mieux illustrer l'entêtement de l'avare, on constate que Séraphin, qui convoite les vaches de Lemont, réussit à les obtenir après mille ruses. Quant à l'avarice, elle est évidence dans tout le roman, et plus particulièrement dans le passage où Séraphin se demande avec inquiétude s'il doit faire venir le docteur de Sainte-Agathe, puisque celui du village est absent: "Tantôt, il se réjouissait d'avoir sauvé deux piastres, tantôt, il souffrait terriblement à la pensée que ça pourrait bien lui en coûter dix. Quelle épreuve, et bien pire que la maladie de Donalda." (p. 88)

Gérard Bessette avait émis l'opinion que les deux cousins soient “complémentaires ${ }^{27}$ " parce que l'un aime l'argent et l'autre est prodigue. En réalité, ils sont complémentaires parce qu'ils sont restés tous deux au stade anal.

25. Sandor Ferenczi, op. cit., p. 143.

26. Ibid., p. 147, note I.

27. Gérard Bessette, op. cit., p. 101. 
Au terme de cette analyse, nous pouvons conclure à une fixation de Séraphin aux stades prégénitaux. Nous avons démontré que la luxure de Séraphin est caractéristique du stade oral: désir d'engloutir, de posséder brutalement des femmes qui représentent la Mère. Son avarice, caractérisée par la rétention de l'argent, et du plaisir qu'il en tire, s'apparente au stade anal de la libido.

En fait, le problème de Séraphin est analogue à celui des autres personnages. Ceux-ci, exception faite de l'avare, souffrent tous d'une fixation qu'on pourrait situer à la période œdipienne. Ceci contribue à créer ce que Bessette a appelé «un climat incestueux ${ }^{28}$ ». Bertine a une admiration fortement incestueuse pour son père: «Bertine comparaît inconsciemment son père, fort, beau et grand, avec Séraphin, doucereux, hésitant, chafouin. Et comme elle l'admira! II partit comme une tempête! Il avait déjà disparu derrière la colline que le regard ardent de sa fille le suivait toujours. " (p. 87)

Quant à Donalda et Alexis, ils manifestent aussi des tendances «incestueuses ${ }^{29}$ " l'un envers l'autre. Le seul nom d'Alexis rend des couleurs à Donalda malade: «À ce seul nom, la malade ouvrit les yeux, et un sourire presque imperceptible effleura ses lèvres sèches. » (p. 62) Et plus loin, dans son délire, elle laisse échapper des paroles fort révélatrices au sujet de ses véritables sentiments pour lui : "On va se marier à c't'heure que je suis veuve." (p. 101) Alexis, lui, rêve dans le lit où Donalda vient de trépasser: "Plus léger qu'un nuage, il flottait dans l'espace sur un printemps sans fin, au-dessus de la campagne en fleurs, avec Donalda à ses côtés, nu-tête, qui présentait sa bouche de fraise au miel du soleil " (p. 132)

Cette étude, comme son titre l'indique, n'a pas la prétention d'être autre chose qu'une contribution à une éventuelle psychocritique d'Un homme et son péché. Une telle étude devrait, tout en s'inspirant des travaux de Bessette, faire ressortir les fixations prégénitales de Séraphin et, par là, le caractère infantile du moi. En ce sens, le "complexe laitier» dont parle Bessette se rattacherait directement à une fixation au stade oral et ne serait pas consécutif à "un investissement érotique des seins ${ }^{30}$ " comme le prétend Bessette. II faudrait également s'intéresser aux personnages secondaires: Bertine, Alexis, Lemont, Brassard, qui ont trop souvent été négligés par la critique. Nous avons déjà fait ressortir le rôle de double du moi joué par Lemont, l'érotisme anal d'Alexis; Gérard Bessette a décrit le beurrier Brassard comme un autre double de Séraphin. De plus, une étude approfondie de certains détails qui reviennent de façon obsédante, par exemple la connotation sexuelle rattachée aux fraises, serait indispensable pour comprendre les mobiles profonds des "héros» d'Un homme et son péché.

28. Gérard Bessette, op. cit., p. 102.

29. Nous employons "incestueux" dans le sens large du mot: gens qui sont apparentes de près. (Donalda est l'épouse du cousin germain d'Alexis.)

30. Gerard Bessette, op. cit., p. 96. 